\title{
Bispectra of climate cycles show how ice ages are fuelled
}

Diederik Liebrand ${ }^{1}$, Anouk T. M. de Bakker ${ }^{2}$

${ }^{1}$ Center for Marine Environmental Sciences (MARUM), University of Bremen, Bremen, Germany

$5 \quad{ }^{2}$ LIttoral ENvironnement et Sociétés (LIENSs), Université de La Rochelle, La Rochelle, France

Correspondence to: D. Liebrand (diederik@palaeoclimate.science)
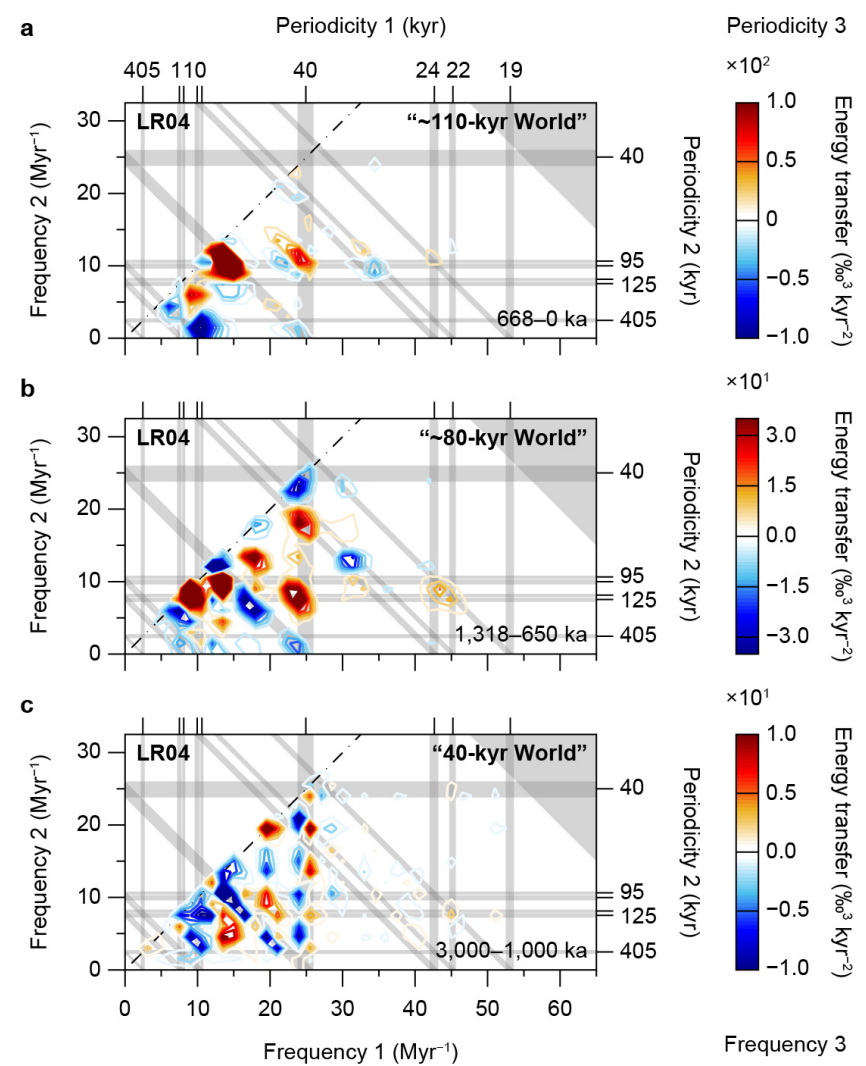

Supplementary Figure S1. Examples of the real part of the bispectrum. These examples correspond to the imaginary parts

10 show in Fig. 4 in the main document. (a) Real part of a bispectrum of the Middle and Late Pleistocene " 110 -kyr world". Min value $=-146 \%{ }^{3} \mathrm{kyr}^{-2}$. Max value $=397 \%^{3} \mathrm{kyr}^{-2}$. (b) Real part of a bispectrum of the mid-Pleistocene transition " $\sim 80$ kyr world". Degrees of freedom $=2$. Min value $=-108 \%{ }^{3} \mathrm{kyr}^{-2}$. Max value $=112 \% \mathrm{kgr}^{3}$. (c) Real part of a bispectrum of the Pliocene and Early Pleistocene "40-kyr world". Min value $=-63 \%^{3} \mathrm{kyr}^{-2}$. Max value $=14 \% \mathrm{kyr}^{3}$. Note the different scaling of the z-axes between panel (a), (b), and (c). Computational settings as in Figure 4 (see main document). These

15 bispectra are computed on the LR04 stack (see Methods) (Lisiecki and Raymo, 2005). 
a

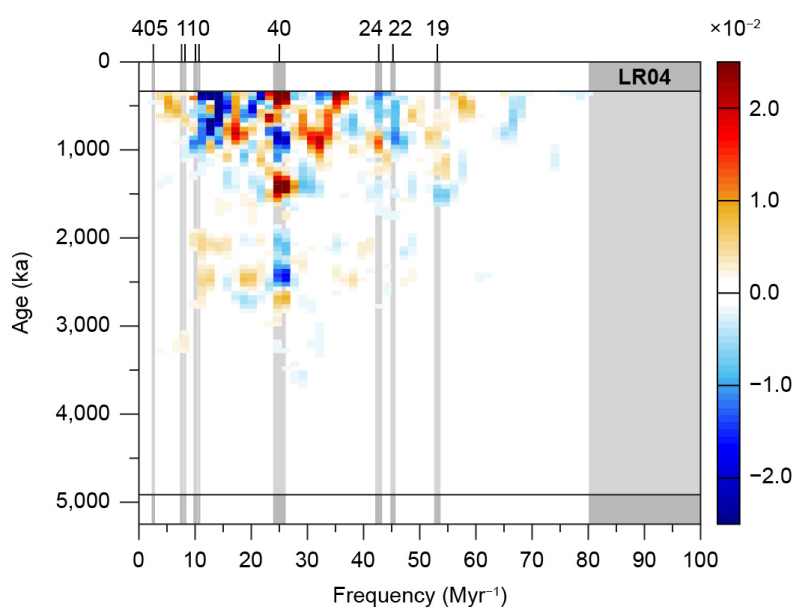

b $\quad 3^{\text {rd }}$ central moment

bispectrum

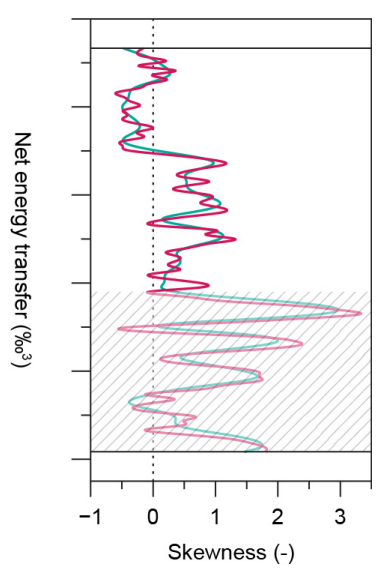

Supplementary Figure S2. Integration over the real part of the bispectrum. This Figure corresponds to Figure $5 \mathrm{~b}$ in the main document. Computational settings as in Figure $5 \mathrm{~b}$ (see main document). 

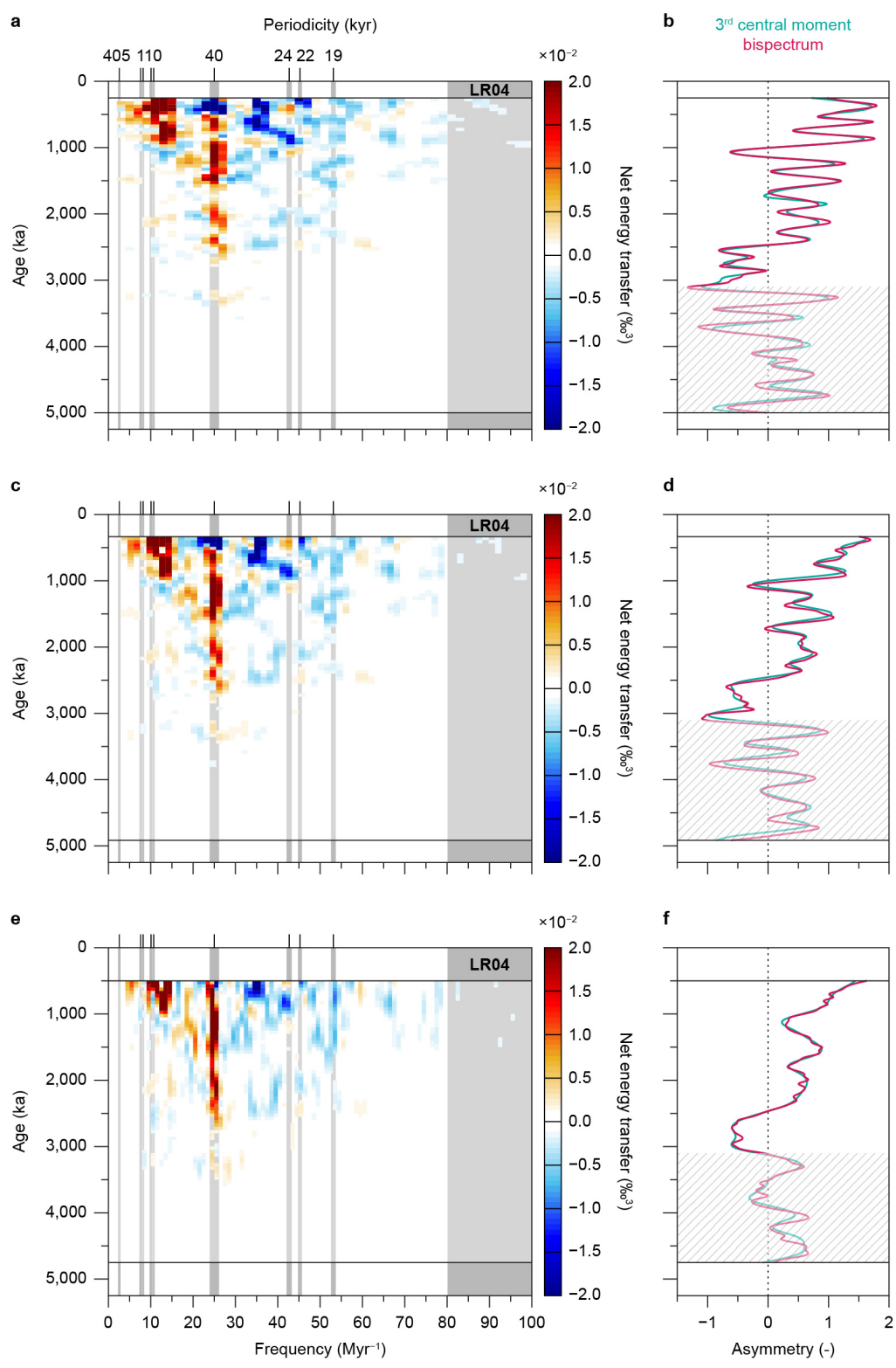

Supplementary Figure S3. Conservative net energy transfers during the Pliocene and Pleistocene, computed by integrating over the entire imaginary part of the bispectrum (see Methods). Input data is the resampled LR04 stack (Lisiecki and Raymo, 2005). (a) Window length $=500$ data points $(500 \mathrm{kyr})$, step-size $=50$ data points $(50 \mathrm{kyr})$. No blocks. No frequency merging.

5 Frequency resolution $=2.00 \mathrm{Myr}^{-1}$. Degrees of freedom $=2$. (b) As in Figure $5 \mathrm{~b}$ (see main document). Window length $=668$ data points $(668 \mathrm{kyr})$, step-size $=50$ data points $(50 \mathrm{kyr})$. No blocks. No frequency merging. Frequency resolution $=1.50$ $\mathrm{Myr}^{-1}$. Degrees of freedom $=2$. (c) Window length $=1,000$ data points $(1,000 \mathrm{kyr})$, step-size $=50$ data points $(50 \mathrm{kyr})$. No blocks. No frequency merging. Frequency resolution $=1.00 \mathrm{Myr}^{-1}$. Degrees of freedom $=2$. 
a. Zone 1: $B^{\prime m}(\mathrm{E}, \mathrm{E}, \mathrm{E})$
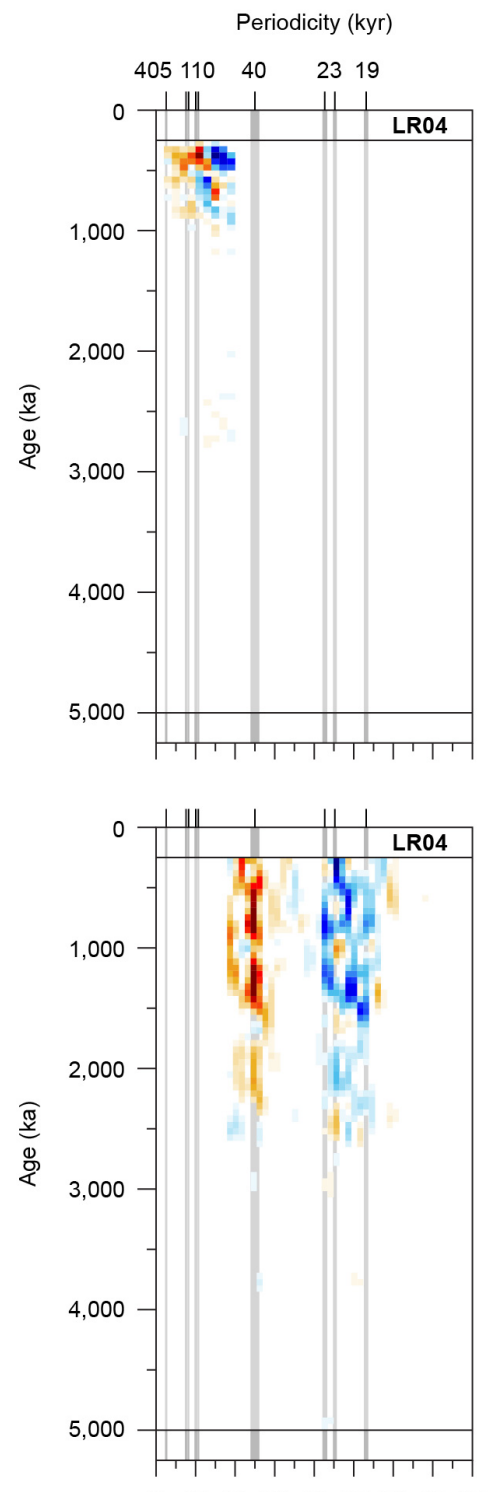

$\begin{array}{lllllllll}0 & 10 & 20 & 30 & 40 & 50 \quad 60 & 70 \quad 80\end{array}$ Frequency $\left(\mathrm{Myr}^{-1}\right)$

e. Zone 5: $B^{l m}(\mathrm{O}, \mathrm{O}, \mathrm{P})$ b. Zone 2: $B^{\operatorname{lm}}(\mathrm{E}, \mathrm{E}, \mathrm{O})$

Periodicity (kyr)
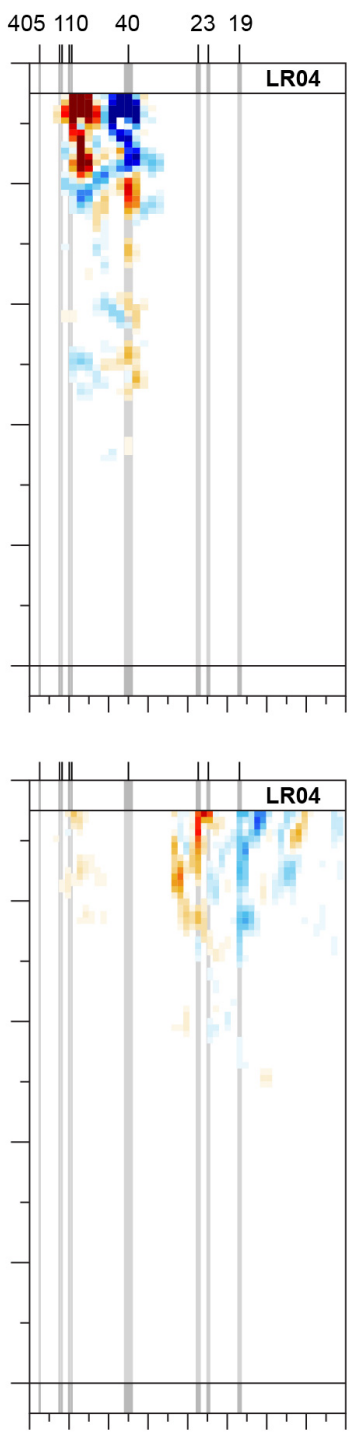

$\begin{array}{lllllllll}0 & 10 & 20 & 30 & 40 & 50 & 60 & 70 & 80\end{array}$ Frequency $\left(\mathrm{Myr}^{-1}\right)$

f. Zone 6: $B^{\prime m}(P, E, P)$ c. Zone 3: $B^{\operatorname{lm}}(\mathrm{O}, \mathrm{E}, \mathrm{O})$

Periodicity (kyr)
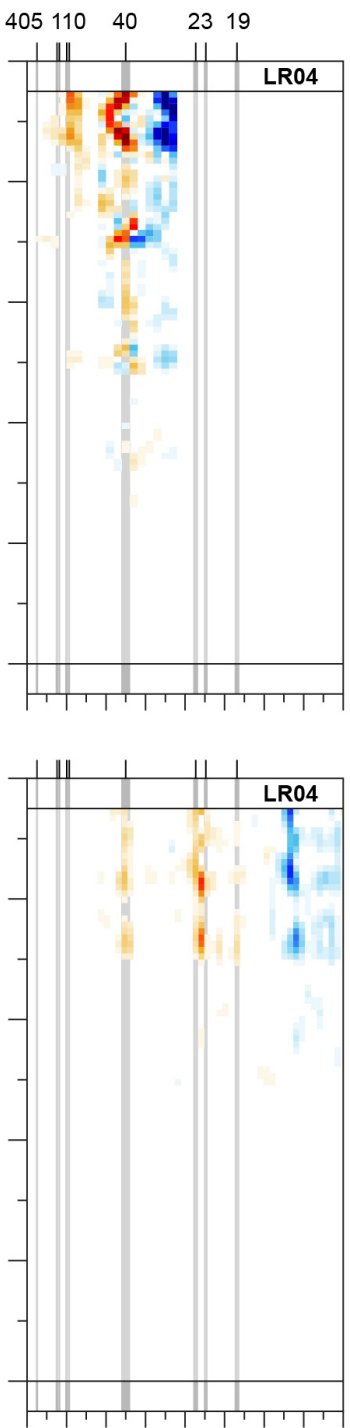

$\begin{array}{lllllllll}0 & 10 & 20 & 30 & 40 & 50 \quad 60 \quad 70 \quad 80\end{array}$

Frequency $\left(\mathrm{Myr}^{-1}\right)$

g. Zone 7: $B^{\prime m}(\mathrm{P}, \mathrm{O}, \mathrm{P})$ d. Zone 4: $B^{I m}(\mathrm{O}, \mathrm{E}, \mathrm{P})$

Periodicity (kyr)
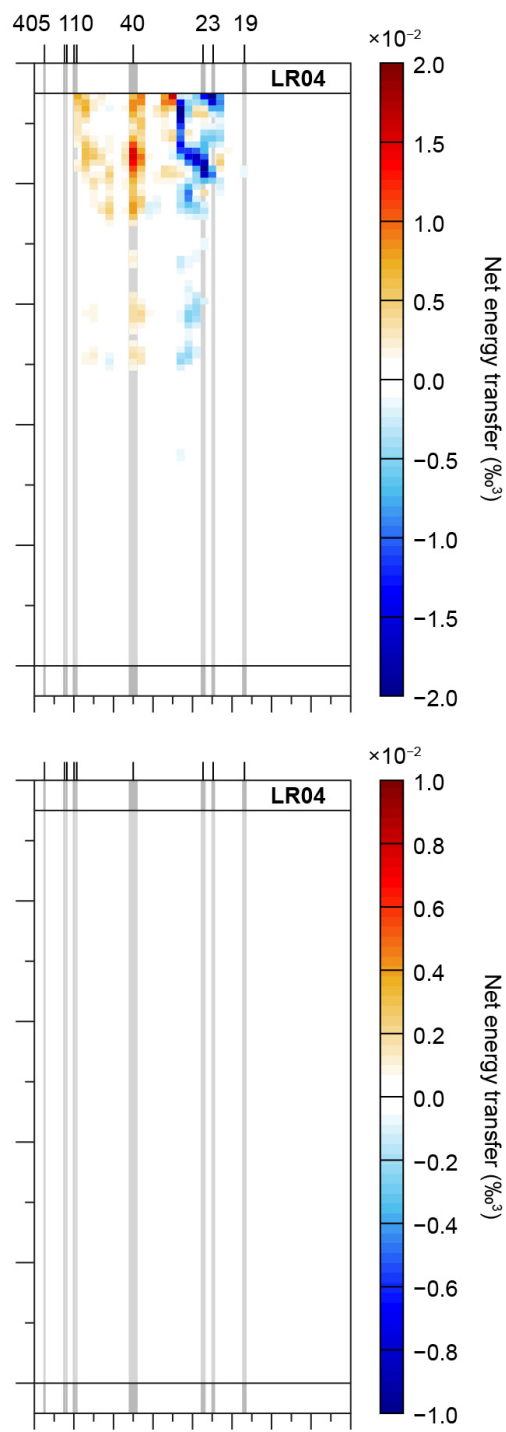

$\begin{array}{lllllllll}0 & 10 & 20 & 30 \quad 40 & 50 \quad 60 \quad 70 \quad 80\end{array}$

Frequency $\left(\mathrm{Myr}^{-1}\right)$

h. Zone 8: $B^{\prime m}(\mathrm{P}, \mathrm{P}, \mathrm{P})$

Supplementary Figure S4. Conservative net energy transfers during the Pliocene and Pleistocene over specific zones in the imaginary part of the bispectrum (see Methods). Computational settings as in Figure S3a (i.e., window length $=500$ data

5 points). (a) Zone 1. (b) Zone 2. (c) Zone 3. (d) Zone 4. (e) Zone 5. (f) Zone 6. (g) Zone 7. (h) Zone 8. See also Figure 3 and Table A1 (main document). 
a. Zone 1: $B^{\prime m}(E, E, E)$
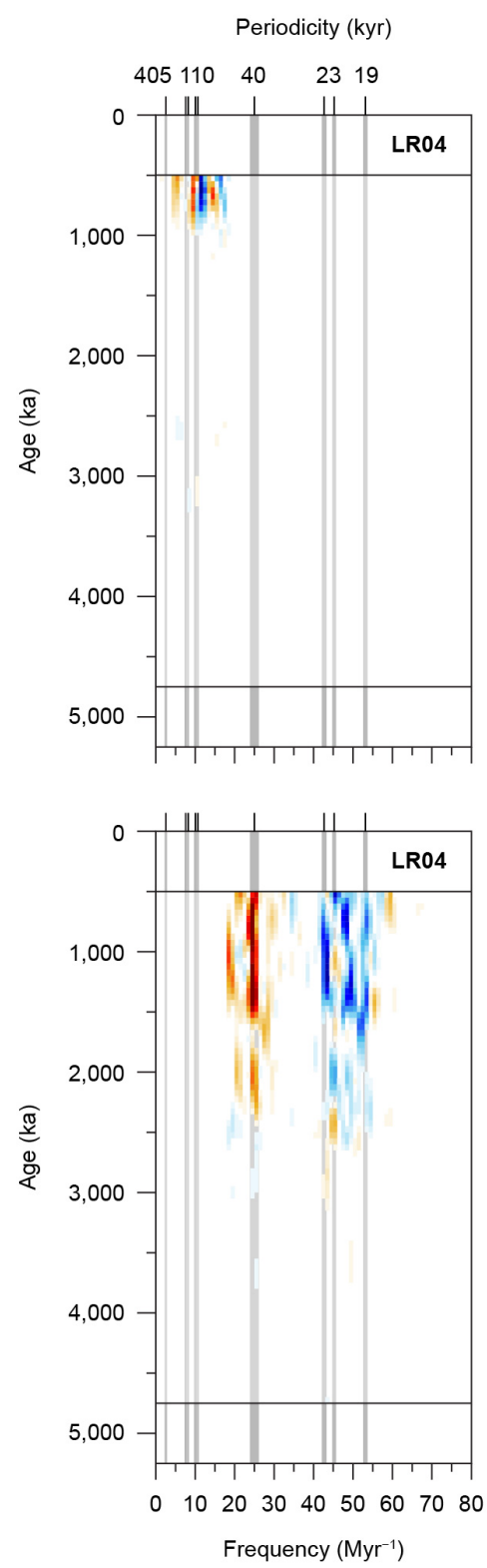

e. Zone 5: $B^{l m}(\mathrm{O}, \mathrm{O}, \mathrm{P})$ b. Zone 2: $B^{\operatorname{lm}}(\mathrm{E}, \mathrm{E}, \mathrm{O})$

Periodicity (kyr)
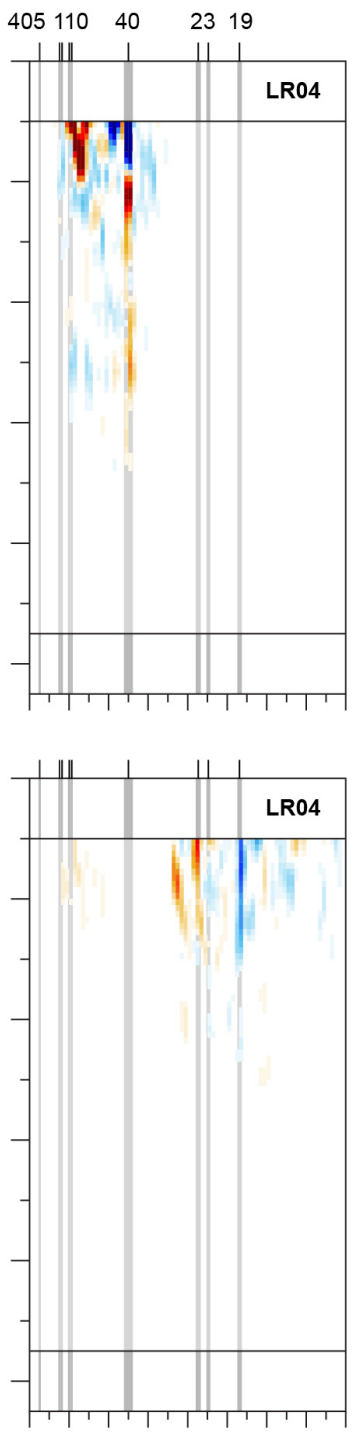

$\begin{array}{llllllll}0 & 10 & 20 & 30 & 40 & 50 \quad 60 & 70 \quad 80\end{array}$ Frequency $\left(\mathrm{Myr}^{-1}\right)$

f. Zone 6: $B^{\prime m}(P, E, P)$ c. Zone 3: $B^{\operatorname{lm}}(\mathrm{O}, \mathrm{E}, \mathrm{O})$

Periodicity (kyr)
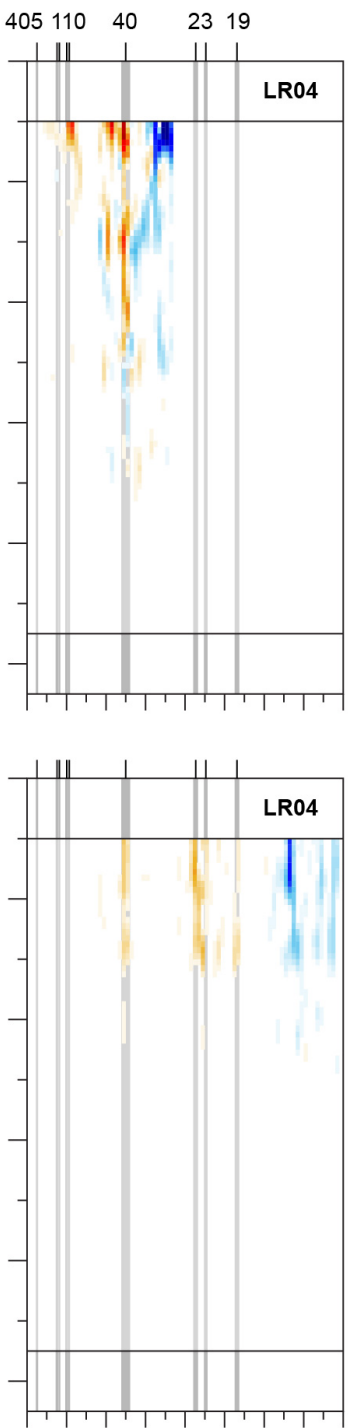

$\begin{array}{lllllllll}0 & 10 & 20 & 30 & 40 & 50 \quad 60 \quad 70 \quad 80\end{array}$ Frequency $\left(\mathrm{Myr}^{-1}\right)$

g. Zone 7: $B^{\prime m}(\mathrm{P}, \mathrm{O}, \mathrm{P})$ d. Zone 4: $B^{I m}(\mathrm{O}, \mathrm{E}, \mathrm{P})$

Periodicity (kyr)
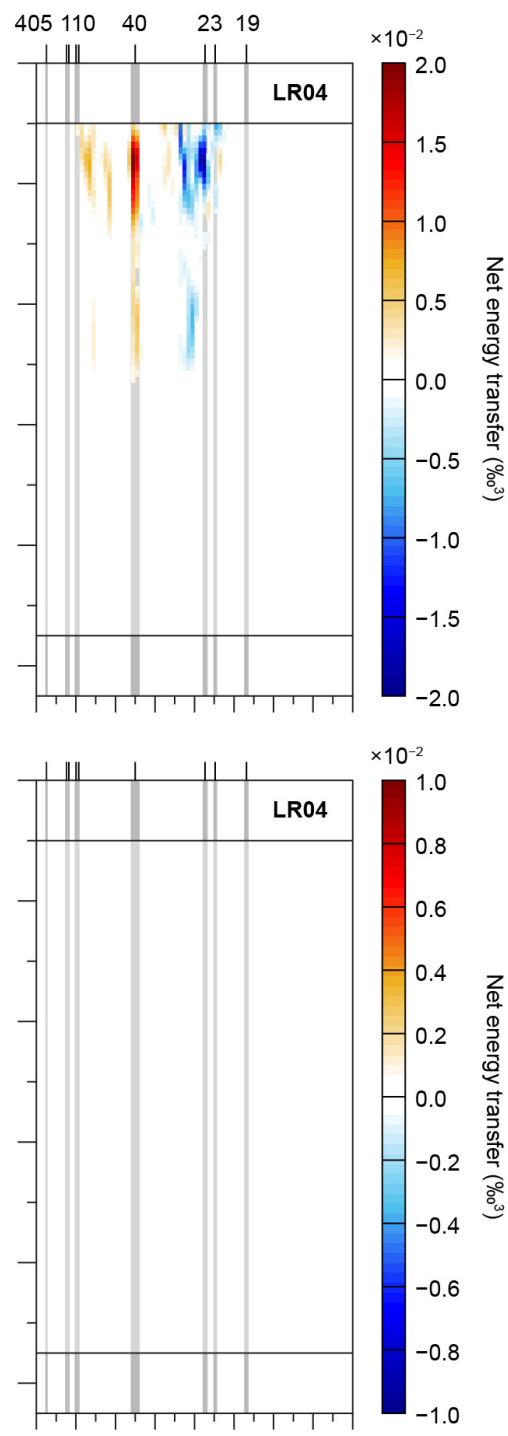

o $10 \quad 20 \quad 30 \quad 40 \quad 50 \quad 60 \quad 70 \quad 80$

Frequency $\left(\mathrm{Myr}^{-1}\right)$

h. Zone 8: $B^{\prime m}(\mathrm{P}, \mathrm{P}, \mathrm{P})$

Supplementary Figure S5. Conservative net energy transfers during the Pliocene and Pleistocene over specific zones in the imaginary part of the bispectrum (see Methods). Computational settings as in Figure S3c (i.e., window length $=1,000$ data

5 points). (a) Zone 1. (b) Zone 2. (c) Zone 3. (d) Zone 4. (e) Zone 5. (f) Zone 6. (g) Zone 7. (h) Zone 8. See also Figure 3 and Table A1 (main document). 


\section{References}

Lisiecki, L. E., and Raymo, M. E.: A Pliocene-Pleistocene stack of 57 globally distributed benthic ${ }^{18} \mathrm{O}$ records, Paleoceanography, 20, https://doi.org/10.1029/2004PA001071, 2005. 